\title{
AN EFFICIENT AlgORITHMiC APPROACH TO THE DESIGN OF SAW COUPLED RESONATOR FILTERS FOR DESIGN AUTOMATION
}

\author{
M. J. McCOLLISTER and S. M. RICHIE \\ University of Central Florida, School of Electrical Engineering and Computer Science, Orlando, Florida 32816
}

Abstract - Design curves have been developed from the previous body of work concerning the design of SAW coupled resonator filters. These curves permit the investigation of design parameters versus filter performance relationships. When introduced into a closed loop design system, these design curves form the core capability of a design automation system. Specifications such as center frequency, insertion loss, bandwidth, group delay, and phase characteristics are input parameters into the algorithm set. The numerical algorithms presented in this paper, in conjunction with a set of design automation algorithms and verification algorithms, combine to form an automated SAW design system that will aid a SAW design engineer in rapid design development. Tradeoffs of accuracy versus execution times are studied. Since a design automation system requires many design iterations to converge to an acceptable solution set, efficient algorithmic approaches are required. The algorithms and programs developed in this paper will be available via the Internet. The algorithms and programs developed in this paper will be available via the Internet at:

http://pegasus.cc.ucf.edu/ mjm05082/ultra2000/.

\section{INTRODUCTION}

The development of a SAW design automation system includes four main parts. These parts are the user specification, rule and model based SAW design, analysis and layout, and performance evaluation and verification [1]. The verification phase has been developed [2] and is used in the algorithms presented here, to measure synthesized device parameters. Of the many SAW devices currently used the coupled resonator filter was chosen for development of design algorithms due to its wide use in industry. The modeling approaches used in development of the design algorithms are the standard coupling of modes models in the current literature [3, 4].

The approach used was to first parameterize oneport resonator performance as a function of structure and then to apply these results to two-port coupled resonator filters using the simple coupling models of Datta [5]. Generic material parameters were used as cited in Morgan [6].

\section{DESIGN CURVE DEVELOPMENT}

In order to quantify the behavioral characteristics of a coupled resonator filter, as shown in Figure 1, a study of resonant structures must be performed. This was done by analyzing a one-port resonator, as shown in Figure 2, to empirically determine the effects each structural variable has on the performance of the device. Values such as center frequency, bandwidth, and minimum insertion loss were extracted as functions of device structure variables.

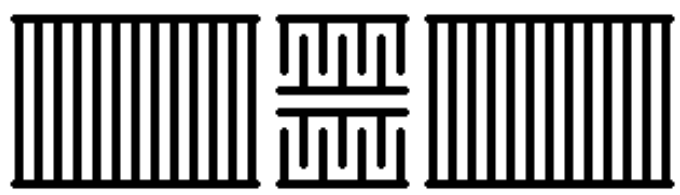

Figure 1: Coupled Resonator

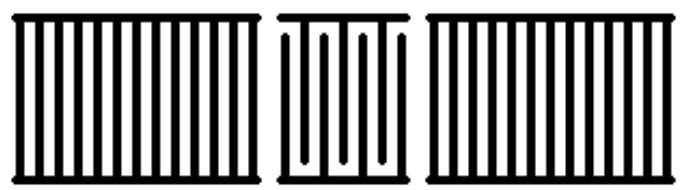

Figure 2: One-Port Resonator

Analyzing the one-port resonator reduces the number of variables in the device structure so that resonant behavior can be more easily modeled. The parameters investigated include the number of grating electrodes, the number of IDT electrodes, the spacing between the gratings (independent of the size of the IDT), the width of the structure, and the metal thickness. The grating and IDT electrode period is used to modify the resonant or center frequency, which is grossly obtained by

$$
p=\frac{T}{2} v_{f} \text { where } T=\frac{1}{f_{0}} .
$$


The investigated one-port resonator device has the default characteristics as show in Table 1. This resonator will resonate at around $150 \mathrm{MHz}$. Parameters that are modified are in italicized in Table 1. Note that the gratings are shorted.

\begin{tabular}{|l|l|}
\hline \multicolumn{2}{|c|}{ Parameter Constant Parameters } \\
\hline \multicolumn{2}{|c|}{ Value } \\
\hline Substrate & ST Quartz \\
\hline Grating and IDT Metalization Ratio & $50 \%$ \\
\hline Resistivity (Rs) & $0.2 \Omega / \mathrm{sq}$ \\
\hline External Load (Zo) & $50 \Omega$ \\
\hline \multicolumn{2}{|c|}{ Modified Parameters } \\
\hline Number of Grating Electrodes & 650 \\
\hline Number of IDT Electrodes & 45 \\
\hline Grating and IDT Electrode Period & $10.5 \mu \mathrm{m}$ \\
\hline Electrode Thickness & $1,800 \AA$ \\
\hline Transducer Width & $1,260 \mu \mathrm{m}$ \\
\hline Separation Between Grating \& IDT & $13.125 \mu \mathrm{m}$ \\
\hline
\end{tabular}

Table 1: Initial One-Port Resonator Values

The models used to analyze a one-port resonator and coupled resonator filter are the standard coupling of modes models. In the analyses presented in this paper, the attenuation factor, and electrode resistance are excluded.

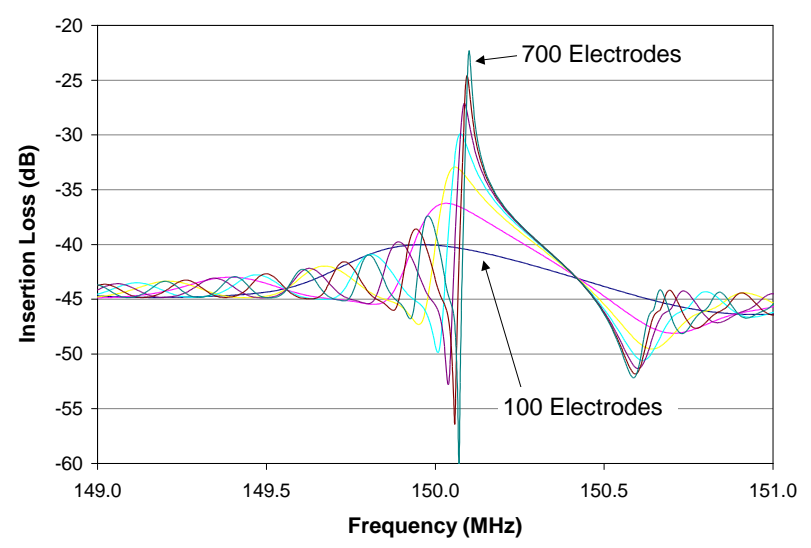

Figure 3: Insertion Loss with Varying Grating Electrodes

The first parameter investigated was the number of grating electrodes. Figure 3 shows the insertion loss $\left(S_{21}\right)$ for 100 to 700 electrodes. For the case of 100 electrodes, the frequency at minimum insertion loss is shifted significantly from the structural frequency as well as having a large insertion. Increasing the number of electrodes reduces the insertion loss and shifts the resonant frequency.
By extracting the minimum insertion loss and the frequency at minimum insertion loss over a range of grating electrodes, a design curve is obtained, as shown in Figure 4.

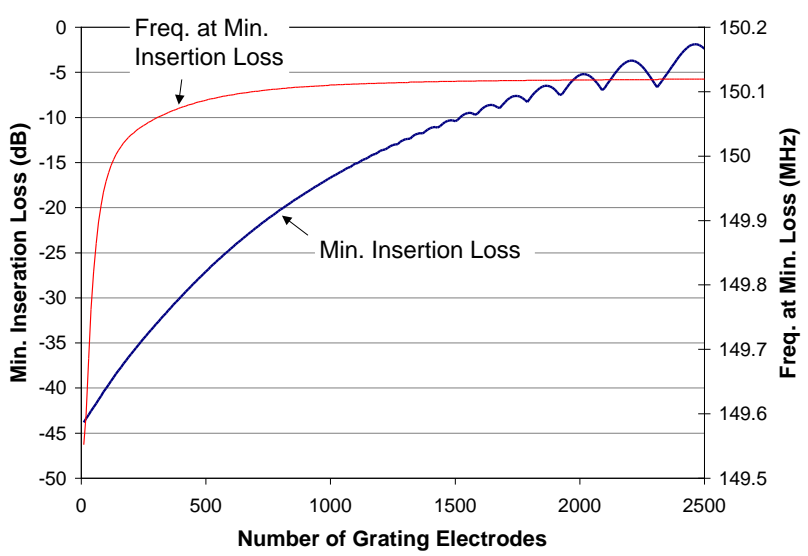

Figure 4: Min. Insertion Loss and Frequency with Varying Grating Electrodes

As shown in Figure 5, varying the number of IDT electrodes, while retaining a constant distance between gratings, the minimum insertion loss increases with the number of electrodes. However, the frequency peaks in the range of 60 to 120 IDT electrodes.

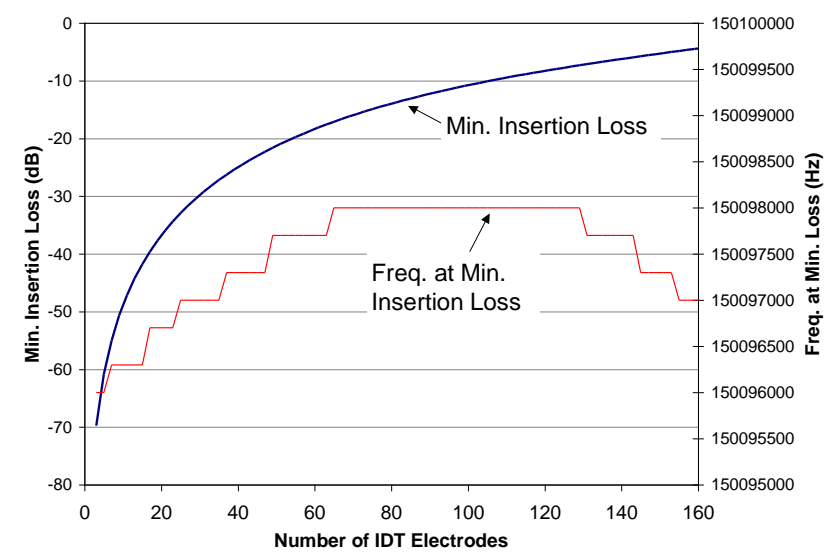

Figure 5: Min. Insertion Loss and Frequency with Varying IDT Electrodes (333 Hz Frequency Resolution)

In Figure 5, the center frequency is highly quantized due to the sampling frequency of the data. The data for this set was run between $150 \mathrm{MHz}$ and $151 \mathrm{MHz}$ with 3,000 sampled points. This resulted in a frequency resolution of $333 \mathrm{~Hz}$, which is shown in Figure 5. However, to get a better approximation of 
the center frequency, more points should be used. By increasing the number of points to 30,000 over the same $1 \mathrm{MHz}$ range, the frequency resolution becomes $33 \mathrm{~Hz}$, but at the cost of processing time. To generate the $333 \mathrm{~Hz}$ resolution data in Figure 5 it took 41 seconds on an $800 \mathrm{MHz}$ Intel Pentium III. To generate the $33 \mathrm{~Hz}$ resolution data, as shown in Figure 6, on the same machine it took 411 seconds, ten time the amount of time. In this case, the accuracy of the center frequency in proportional to the computing time required. This knowledge is used to efficiently converge any automated design iterations.

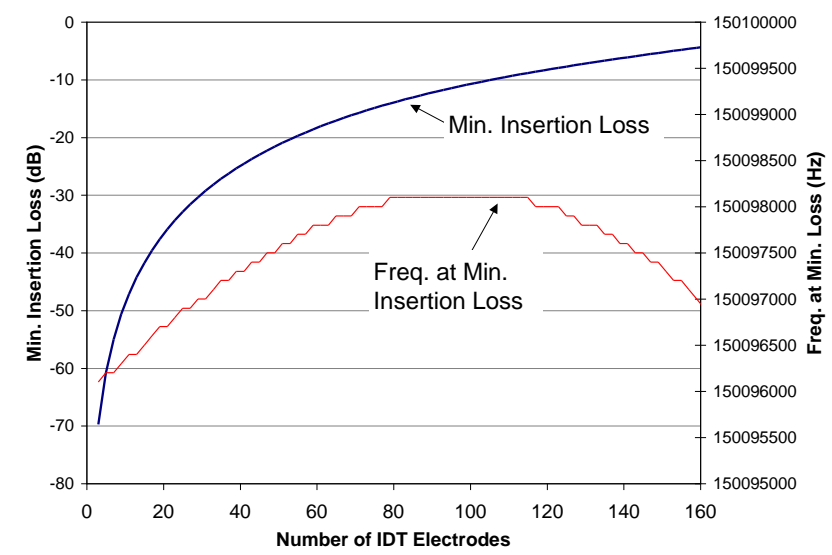

Figure 6: Min. Insertion Loss and Frequency with Varying IDT Electrodes (33 Hz Frequency Resolution)

In an intelligent design automation system the initial analysis is performed at a lower resolution so that a course response can be quickly obtained. The course response is then used as the basis for a second phase, which is performed at a higher resolution to get the desired highly accurate results.

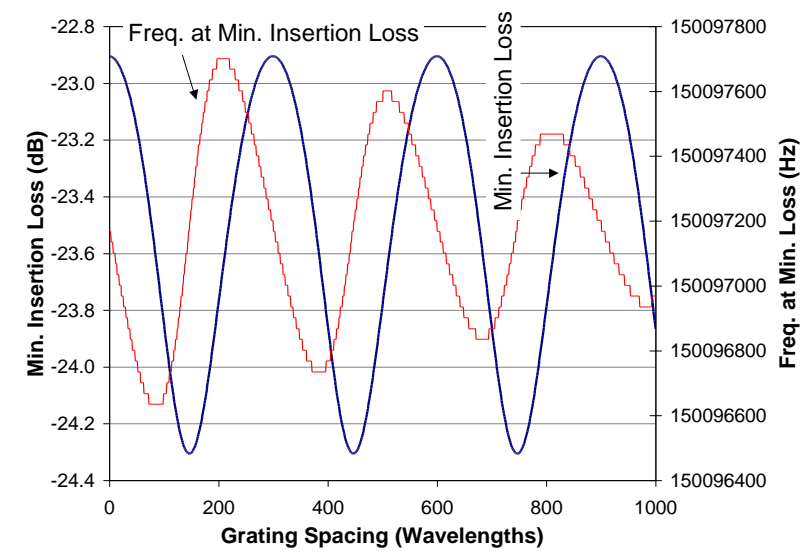

Figure 7: Min. Insertion Loss and Frequency with Varying Grating Spacing
By varying the grating spacing, in wavelengths, the minimum insertion loss varies by $1.4 \mathrm{~dB}$ in a sinusoidal fashion, as shown in Figure 7. The frequency varies in a damped sinusoidal fashion over a range of approximately $1 \mathrm{KHz}$. Again, the frequency is highly quantized due to the $33 \mathrm{~Hz}$ resolution of the sampled data. The sinusoidal nature of the minimum insertion loss indicates that it is desirable to pick a peak value.

Varying the width of the grating and IDT, results in a significant swing in the minimum insertion loss, as shown in Figure 8. Since it approaches, $-3 \mathrm{~dB}$ asymptotically, there is no reason to select a structure width more than is necessary. Figure 8 also shows that the $33 \mathrm{~Hz}$ resolution center frequency decreases as the width of the structure increases.

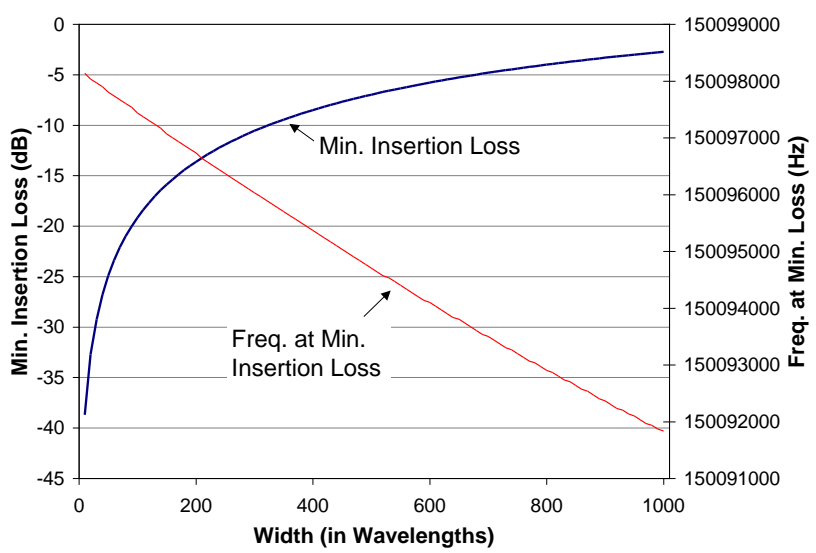

Figure 8: Min. Insertion Loss \& Freq. with Varying Width

Figure 9 shows that the metal thickness of the grating and IDT electrodes is also a major contributor

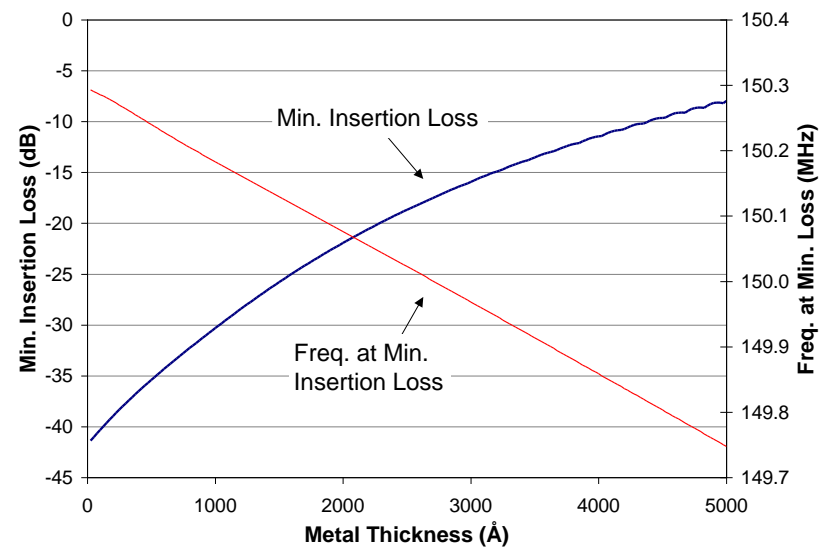

Figure 9: Min. Insertion Loss and Frequency with Varying Metal Thickness 
to the minimum insertion loss. However, the center frequency is highly dependant on the metal thickness and varies by as much as $500 \mathrm{KHz}$ over the range of $25 \AA$ to $5000 \AA$.

The analyses run on the one-port resonator structure are now used in the design of couple resonator filters as discussed below.

\section{DESIGN ALGORITHMS}

By curve fitting the data from the previous section to the best functions, predictive equations were obtained. For Number of Grating Electrodes (where $N_{g}$ is the number of grating electrodes):

$$
\begin{gathered}
\left|S_{21}\right|_{\text {min- } \mathrm{dB}}=\begin{array}{l}
2.5 \times 10^{-9} N_{g}{ }^{3}-16 \times 10^{-6} N_{g}{ }^{2}+ \\
0.0408 N_{g}-43.9
\end{array} \\
f_{0}=24.6 N_{g}+150.1 \times 10^{6}-7354199 N_{g}{ }^{-1}
\end{gathered}
$$

For Number of IDT Electrodes (where $N_{t}$ is the number of IDT electrodes):

$$
\begin{gathered}
\left|S_{21}\right|_{\min -\mathrm{dB}}=-\exp \left(-0.014 N_{t}+3.75+1.96 N_{t}^{-1}\right), \\
f_{0}=-0.25 N_{t}^{2}+47.7 N_{t}+150.1 \times 10^{6} .
\end{gathered}
$$

For Grating Spacing (where $D_{m}$ is spacing in wavelengths):

$$
\begin{gathered}
\left|S_{21}\right|_{\text {min }-\mathrm{dB}}=-23.6+0.685 \cos \left(\frac{2 \pi}{300} D_{m}\right), \\
f_{0}=150.1 \times 10^{6}+\left(-0.33 D_{m}+553\right) \cos \left[\frac{2 \pi}{300}\left(D_{m}-213\right)\right] .
\end{gathered}
$$

For Width (where $W$ is width in wavelengths):

$$
\begin{aligned}
& \left|S_{21}\right|_{\text {min-dB }}=-14.47 \times 10^{6} W^{2}+0.028 W-16.41+ \\
& -517.9 W^{-1}+3 W^{-2} \\
& f_{0}=1.2 \times 10^{-3} \mathrm{~W}^{2}-7.54 \mathrm{~W}+150.1 \times 10^{6} .
\end{aligned}
$$

For Metal Thickness (where $H_{m}$ is thickness in angstroms):

$$
\begin{gathered}
\left|S_{21}\right|_{\min -\mathrm{dB}}=-\exp \left(-326 \times 10^{-6} H_{m}+3.7385\right), \\
f_{0}=-78.2 \times 10^{-6}{H_{m}}^{2}-108.6 H_{m}+150.3 \times 10^{6} .
\end{gathered}
$$

\section{CONCLUSIONS}

The design algorithms presented in this paper have been created using an ANSI C++ program called AutoSAW. AutoSAW is highly object oriented and uses a component based architecture. The components start from a basic SAW class which defines and holds parameters that are common to all SAW structures. The grating and IDT structures inherit the SAW base class. The one-port resonator inherits the SAW base and includes two gratings and one IDT. The coupled resonator, the highest level, inherits properties of the one-port resonator and builds upon its methods, as shown in Figure 10. Using the object oriented approach has simplified the model so that it is very easy modify any of the device parameters and quickly get the response of the device. Also, the object-oriented approach makes it easy to quickly change the device equations, such as the coupling of modes model.

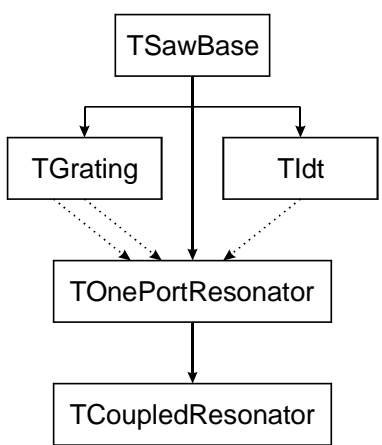

Figure 10: Inheritance Diagram of SAW Resonator Models

\section{REFERENCES}

[1] M. J. McCollister and S. M. Richie, "The Development of a First Pass Verification Module for a SAW Filter Design Automation System", IEEE Ultrasonics Symposium Proceedings, vol. 1, pp. 133-136, 1991.

[2] M. J. McCollister and S. M. Richie, "The Design Verification Module for a SAW Design Automation System," 1999 Frequency Control Symposium Proceedings, 1999, vol. 2, pp. 891-894.

[3] T. Thorvaldsson, "Design and Analysis of Surface Acoustic Wave Resonator and Resonator Filters," Ph. D. Dissertation, University of Central Florida, Orlando Florida, 1986.

[4] B. P. Abbott, "A Coupling-of-Modes Model for SAW Transducers with Arbitrary Reflectivity Weighting," Ph. D. Dissertation, University of Central Florida, Orlando Florida, 1986.

[5] S. Datta, Surface Acoustic Wave Devices, New Jersey: Prentice-Hall, 1986, ch. 8, pp. 187-193.

[6] D. P. Morgan, Surface-Wave Devices for Signal Processing, New York: Elsevier, 1985. 